\title{
A graph coloring-based interference coordination algorithm for cluster-wise ultra-dense RAN
}

\author{
Chang Ge ${ }^{1,2 \text {, a) }}$, Sijie Xia ${ }^{1,2}$, Qiang Chen ${ }^{1}$, and Fumiyuki Adachi ${ }^{2}$ \\ 1 Department of Communications Engineering, Graduate School of Engineering, \\ Tohoku University \\ 6-6-05 Aramaki Aza Aoba, Aoba-ku, Sendai, Miyagi, 980-8579, Japan \\ ${ }^{2}$ Research Organization of Electrical Communication, Tohoku University \\ 2-1-1 Katahira, Aoba-ku, Sendai, Miyagi, 980-8577, Japan \\ a) ge.chang.q2@dc.tohoku.ac.jp
}

Abstract: Ultra-dense radio access network (RAN) with distributed multiuser multi-input multi-output (MU-MIMO) is considered as a promising approach to improve the coverage and link capacity. However, its prohibitively high computational complexity impacts its further extension and application. Our previous work has proved that forming users/antennas clusters can greatly alleviate the computational complexity, but the inter-cluster interference is produced, thereby reducing the spectrum efficiency. In this paper, we propose a graph coloring algorithm-based inter-cluster interference coordination for ultra-dense RAN with distributed MU-MIMO. It is confirmed by computer simulation that our proposed algorithm can improve both the sum capacity and the user capacity compared with no coloring case.

Keywords: interference coordination, graph coloring, distributed antennas, ultra-dense RAN, Delaunay triangulation, K-means clustering

Classification: Wireless Communication Technologies

\section{References}

[1] F. Adachi, R. Takahashi, and H. Matsuo, "Enhanced interference coordination and radio resource management for 5G advanced ultra-dense RAN," Proc. The 2020 IEEE 91st Vehicular Technology Conference (VTC2020-Spring): Technology Trials and Proof-of-Concept Activities for 5G Evolution \& Beyond 5G 2020 (TPoC5GE 2020), pp. 1-5, 25-28 May, 2020. DOI: 10.1109/VTC2020Spring48590.2020.9128516

[2] C. Ge, S. Xia, Q. Chen, and F. Adachi, "2-steps graph coloring algorithm for interference coordination in 5G advanced ultra-dense RAN," IEICE Technical Report, RCS2020-13, pp. 19-24, May 2020.

[3] J. Zheng, D. Jia, and B. Zhang, "A two-tier clustering based downlink resource allocation algorithm for small cell networks," Proc. 2019 15th International Wireless Communication \& Mobile Computing Conference (IWCMC), Tangier, Morocco, Morocco, pp. 180-185, 24-28 June 2019. DOI: 10.1109/IWCMC. 2019.8766562 
[4] Y. Wu, H. Xia, Y. Lu, C. Feng, T. Zhang, R. Han, and H. Zhou, "Clustering-based time-domain inter-cell interference coordination in dense small cell networks," Proc. 2014 IEEE 25th Annual International Symposium on Personal, Indoor, and Mobile Radio Communication (PIMRC), Washington, DC, USA, pp. 570-574, 2-5 Sept. 2014. DOI: 10.1109/PIMRC.2014.7136230

[5] J. Keil and C. Gutwin, "Classes of graphs which approximate the complete Euclidean graph," Discrete and Computational Geometry, vol. 7, no. 1, pp. 1328, 1992. DOI: 10.1007/BF02187821

[6] C. Ge, S. Xia, Q. Chen, and F. Adachi, "The influence of different clustering method on graph coloring algorithm in ultra-dense RAN," Proc. IEICE Society Conference 2020, online, 15-18 Sept. 2020.

\section{Introduction}

The millimeter wave band is utilized in $5 \mathrm{G}$ systems due to the shortage of available radio bandwidth, but its rectilinear propagation nature easily causes the occurrences of radio link blockage. In order to improve the coverage and link capacity, the ultradense radio access network (RAN) with distributed MU-MIMO is highly expected. However, a large-scale MU-MIMO, which deals with a large number of users and antennas, has a problem of high computational complexity and therefore, there is a need to find a computationally efficient solution [1].

In our previous study, we proposed a 2-layer clustering algorithm that can group nearby users and antennas into clusters in each base station (BS) coverage area through K-means algorithm [2]. Through this way, the large-scale MU-MIMO can be divided into several cluster-wise small-scale MU-MIMOs, and the computational complexity can be reduced to a practical level. Besides that, the same frequency will be reused in clusters, so the spectrum efficiency will be improved. In each cluster, the zero-forcing (ZF) based cluster-wise MU-MIMO is performed, so the interference inside each cluster can be eliminated. However, the introducing of clusters will bring in a new severe interference between clusters, named inter-cluster interference (ICI), which may offset the spectrum efficiency improvement. Therefore, interference coordination method aimed at mitigating the ICI is also required.

In the similar small cell networks, where the interference coordination is also highly required, one promising idea is to apply graph coloring algorithm to allocate different colors (equivalent to allocate different frequencies) to the small cells to avoid strong interference. Similar to [3] and [4], graph coloring algorithm can be used to divide the small cells into different color groups so as to avoid sharing the same frequency among those who are close to each other.

In this paper, we will introduce graph coloring algorithm into ultra-dense RAN with distributed MU-MIMO, and propose an interference coordination algorithm named restricted color number (RCN) algorithm. Here, we utilize Delaunay triangulation [5] to decide the interference graph from the view of graphics instead of setting threshold in advance. Also, we restrict the maximum number of colors to a certain value in order to improve the utilization of the limited bandwidth.

The rest of this paper is organized as follows. The system model and our proposed RCN algorithm is described in Section 2. The link capacity is evaluated by computer 
simulation in Section 3. Section 4 offers the conclusion and future plan.

\section{Graph coloring-based interference coordination algorithm}

Figure 1 (a) illustrates the sketch graph of our system. For computer simulation, we assume a normalized square-shaped BS area of 1 by 1 in which 128 antennas and single-antenna users are randomly located, one case of 96 users are shown in Fig. 1 (b). In [6], we have already proved that the user-based clustering method jointly used with graph coloring is less affected by the antennas' location and can provide more stable result than antenna-based clustering method. Therefore 2layer (user-based) clustering algorithm is applied in this paper, and the 8 clusters' clustering results for Fig. 1 (b) is shown in Fig. 1 (c).

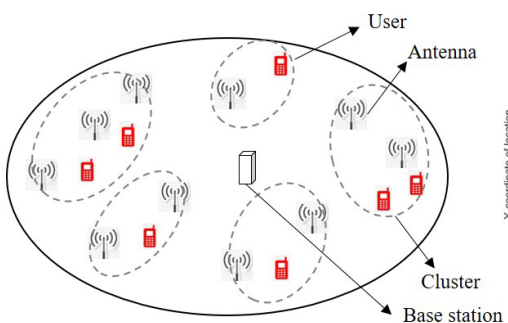

Fig.1.(a) Sketch graph of ultra-dense RAN with distributed cluster-wise MUMIMO

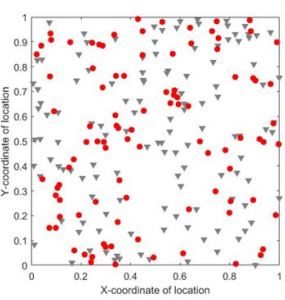

Fig.1.(b) system model for computer simulation

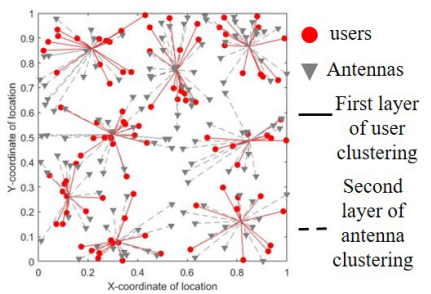

Fig.1.(c) clustering results based on 2-layer clustering algorithm

Fig. 1. The system model description and the clustering results for 96 users, 128 antennas and 8 clusters

Once the clustering results is obtained, our proposed $\mathrm{RCN}$ algorithm can be applied. Our RCN algorithm consists of 2 steps and its flowchart is illustrated in Fig. 2. The maximum number of colors $(M)$ is set initially so as to make sure that the bandwidth will not be divided into too many narrow parts. The $1^{\text {st }}$ step is the modified graph coloring process which assigns different colors to neighbor clusters to eliminate the severe ICI. The graph coloring algorithm in this step is modified by introducing Delaunay Triangulation (see Sect. 2.1). Since we apply the userbased clustering method, the cluster topology may change according to the change in the users' location in each trial. Therefore, the actual number of colors $(A)$ is uncontrollable and may exceed $M$ at some probability. If it happens, the $2^{\text {nd }}$ step of recoloring process (see Sect. 2.2) will be activated. The cluster whose $A$ exceeds $M$ will be recolored as the least interfered cluster, thereby make sure the total colors will not exceed $M$.

\subsection{Modified graph coloring process}

The graph coloring problem can be simplified as an undirected graph $G=(V, E)$, in which $\mathrm{V}$ denotes vertices and $\mathrm{E}$ denotes edges. Since our clustering algorithm ensures there are no overlapping regions among the clusters, $\mathrm{V}$ can be regarded as a set of cluster centroids, while the edges $\mathrm{E}$ always represent the interference relationship.

Before graph coloring, the interference relationship among clusters needs to be decided. It is preferable to introduce the threshold as a criterion to judge whether 


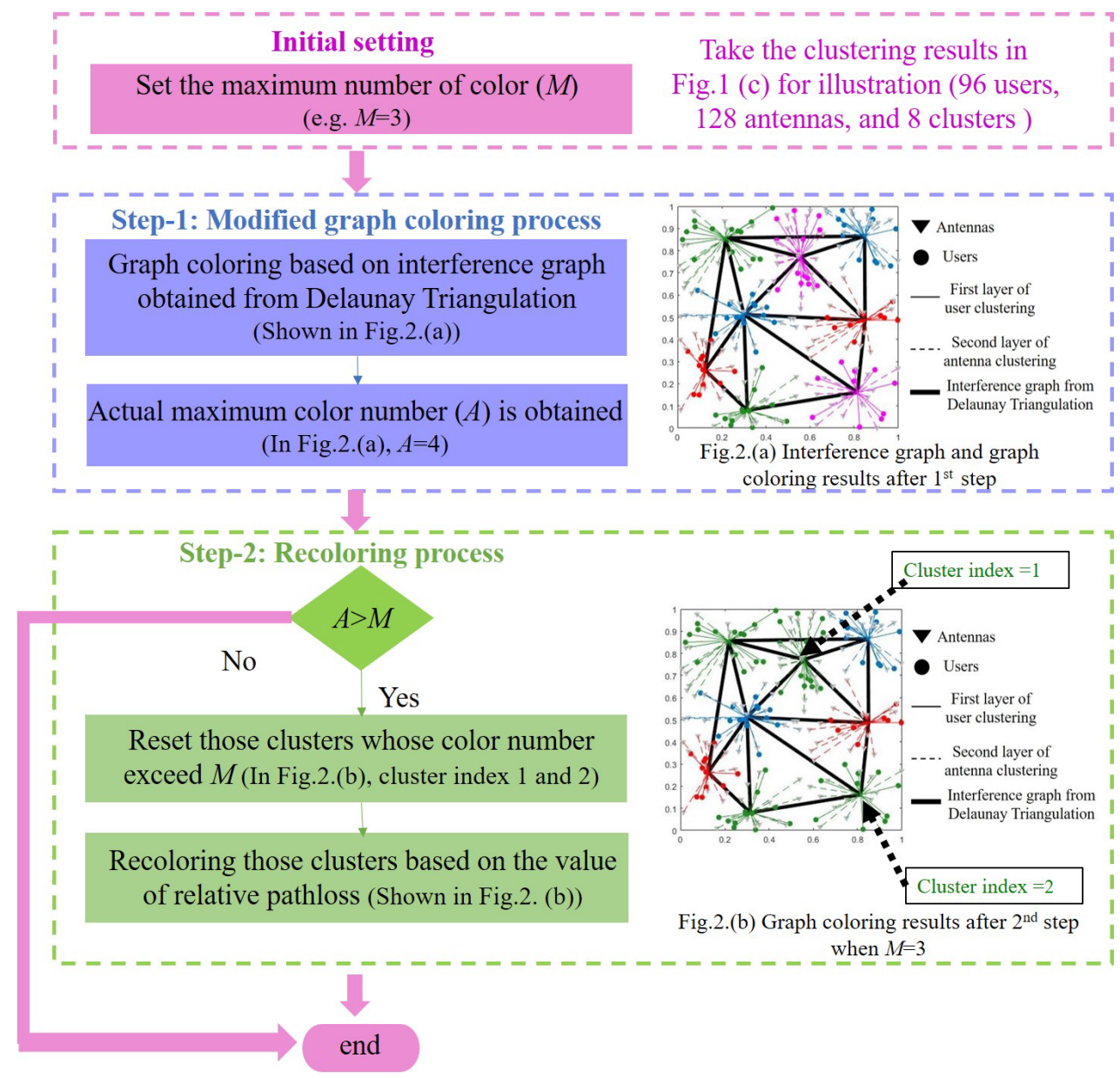

Fig. 2. RCN algorithm

the two clusters are interfered or not. As the threshold, Euclidean distance [3] or interference level [4] can be used. However, how to set the threshold is a problem. Furthermore, the complicated environment of distributed antenna system makes it even tougher to find an optimum threshold value. In our system, the most severe interference is produced between neighboring clusters, thus to a certain extent, the interference relationship can be simplified as the neighboring relationship. In this paper, we introduce Delaunay Triangulation [5] into our algorithm to decide the neighboring relationship from the view of computational geometry and to avoid the use of threshold value. Delaunay triangulation is a way to generate triangles based on a set of discrete vertices that can avoid sliver triangles (a triangle with one or two extremely acute angles). If there is a triangle's edge connecting two centroids, these two clusters will be regarded as neighbors and cannot share the same color. Fig. 2 (a) shows the interference graph and the coloring result for Fig. 1 (c). The value of $A$ equals 4 for this case.

\subsection{Recoloring process}

The $1^{\text {st }}$ step can guarantee a good interference mitigation results since no neighboring clusters share the same color, but the value of $A$ is uncontrollable. The link capacity $[\mathrm{bps} / \mathrm{Hz}]$ is given as 


$$
\text { Capacity }=\frac{1}{A} \log _{2}(1+\operatorname{SINR}) \approx \frac{1}{A} \log _{2}(\operatorname{SINR}) .
$$

The more colors been used, the narrower bandwidth each color group could share, which in turn may lead to a lower link capacity. Therefore, in order to improve the utilization of the entire bandwidth, the maximum number $M$ of colors needs to be restricted.

For the case in Fig. 1 (c), if the initial setting $M=3$, the condition $A>M$ is satisfied and the recoloring process is activated, so the cluster index 1 and 2 need to be recolored. In order to minimize the ICI, the best solution is to choose the least interfered cluster and use its color. Therefore, we introduce a parameter named relative pathloss $D_{i j}$ to search for the least interfered cluster $j$ for cluster index $i$ :

$$
D_{i j}=\frac{d_{i j}^{-\gamma}}{\sum_{j=1, j \neq i}^{N} d_{i j}^{-\gamma}}, i, j=1 \sim N,
$$

where $d_{i j}$ denotes the distance from the cluster $j$ 's antenna's centroid to cluster $i$ 's user's centroid, $N$ is the number of clusters, and $\gamma$ is the pathloss exponent.

\section{Monte Carlo simulation}

In this paper, we evaluate the downlink capacity when cluster-wise MU-MIMO with ZF precoding is used. The antenna locations are kept unchanged (as shown in Fig. 1 (b)), while the users' locations are changed every trial. The transmit power for each user is set so that the received signal-to-noise ratio becomes $0 \mathrm{~dB}$ when the distance between the transmitter and receiver is equal to the side length of square-shaped BS area. The propagation channel is assumed to be composed of the distance-dependent pathloss, log-normal shadowing loss, and Raleigh fading. The pathloss exponent and the shadowing standard deviation are set to 3.5 and $8 \mathrm{~dB}$, respectively.

Figure 2 plots the 50\% sum link capacity and $10 \%$ user capacity as a function of the number of users when the number of clusters equals 8 (note that $\mathrm{x} \%$ capacity is defined as the value of capacity below which the capacity falls at a probability of $x \%$ ). The range of the number of users is from 8 (each cluster has at least one

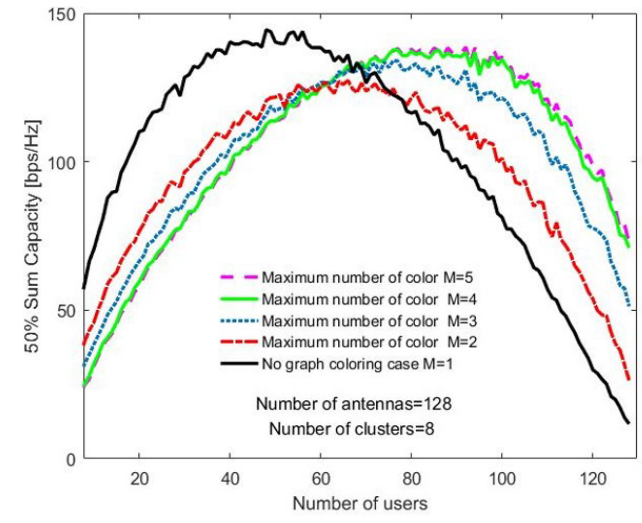

(a) $50 \%$ Sum capacity

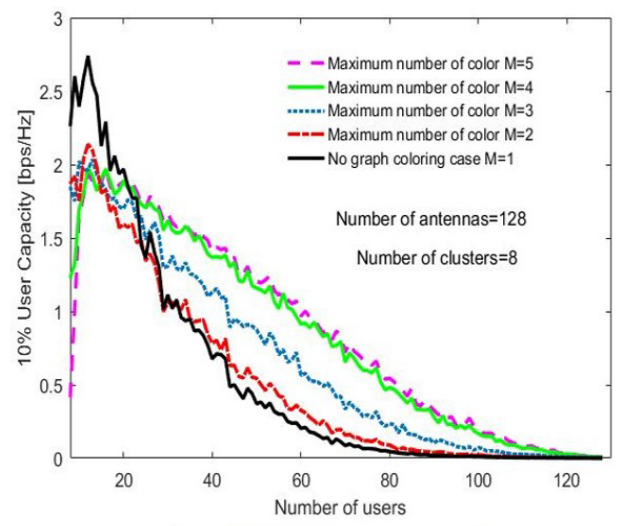

(b) $10 \%$ User capacity
Fig. 3. Comparison of downlink capacity as a function of the number of users. 
user) to 128 (to satisfy the ZF requirement). The link capacity for each number of users is obtained by carrying out 10,000 trials. From the results in Fig. 3, when the number of users is more than half of the number of antennas, the effectiveness of graph coloring algorithm over no coloring case on capacity improvement is obvious.

\section{Conclusion}

In this paper, we verified the effectiveness of graph coloring to mitigate the intercluster interference in ultra-dense RAN with cluster-wise distributed MU-MIMO, and proposed a restricted color number ( $\mathrm{RCN})$ algorithm. We introduced Delaunay triangulation to decide the neighboring relationship from the view of graphics and then described how to restrict the maximum number of colors in order to avoid excess division of the bandwidth. From the link capacity evaluation, we recommend to apply graph coloring algorithm when the number of users is more than half of the number of antennas in practical application.

For future research, we are going to introduce machine learning into our algorithm and expand our research into multicell system.

\section{Acknowledgments}

A part of this work was conducted under " $R \& D$ for further advancement of the $5^{\text {th }}$ generation mobile communication system" (JPJ000254) commissioned by Research and Development for Expansion of Radio Wave Resources of the Ministry of Internal Affairs and Communications in Japan. 